\title{
Análisis de las vibraciones de un motor ciclo OTTO con una mezcla combustible a base de gasolina y de etanol
}

\section{Analysis of the vibrations of an OTTO cycle engine with a fuel mixture based on gasoline and ethanol}

Phd. Marcos Gutiérrez

Tablet School-Escuela de Ciencias y Centro de Investigación Científica, Ecuador

Juan Fernando Iñiguez Izquierdo

Xavier Cadena

Gabriel Santiana

Universidad Internacional del Ecuador, Ecuador

Autor para correspondencia: marcosgutierrez@tablet-school.com,jiniguez@uide.edu.ec Fecha de recepción: 10 de Octubre de 2017 - Fecha de aceptación: 30 de Noviembre de 2017

\section{Resumen}

La presente investigación analiza las vibraciones de un motor ciclo Otto, al combustionar las gasolinas tipo extra y ecopaís, las mismas que son comercializadas en el Ecuador. El motor de prueba se puso en funcionamiento en condiciones estáticas y dinámicas, esta última, por medio de un dinamómetro de rodillos; mientras que las vibraciones fueron registradas por medio de sensores de aceleración de un dispositivo de comunicación móvil, a diferentes velocidades de giro de motor. Los resultados obtenidos muestran que, a pesar de que los combustibles pueden ser utilizados como sustitutos uno del otro, la gasolina del tipo ecopaís constituida por 5\% de etanol y $95 \%$ de gasolina tipo extra, registra un menor nivel de vibración en comparación con la gasolina tipo extra al 100\%. La presente investigación muestra una técnica para el análisis de la estabilidad del motor comparando diferentes tipos de combustibles.

Palabras Clave: vibraciones; motor; gasolina

\begin{abstract}
The present investigation analyzes the vibrations of an Otto cycle engine, by combining the gasolines extra and ecopaís, the same ones that are commercialized in Ecuador. The engine test was performed in static and dynamic conditions with a chassis dynamometer. The vibrations were collected by the acceleration sensors of a mobile communication device, at different engine speeds. The results show that, although the fuels can be used as substitutes for each other, the gasoline type ecopaís consisting of 5\% of ethanol and $95 \%$ of gasoline type extra, registers a lower level of vibration in comparison with the $100 \%$ gasoline type extra. The present research shows a technique for the analysis of the stability of the engine comparing different types of fuels.
\end{abstract}

Key words: vibrations; engine; gasoline 


\section{Introducción}

Con la finalidad de reducir las emisiones contaminantes y la dependencia en las reservas de petróleo a nivel mundial, se han venido utilizando combustibles alternativos como el etanol para el reemplazo total o parcial de la gasolina pura. Mezclas de $85 \%$ de contenido de etanol y mayores, se utilizan en motores diseñados para operar con este tipo de combustible, mientras que mezclas de hasta $10 \%$ de etanol son usadas en motores diseñados para la combustión de gasolina pura. En la actualidad se busca incrementar la concentración de etanol e investigar sus efectos en los motores. [1].

Uno de los efectos que tiene el combustible como consecuencia del proceso de combustión son las vibraciones, además de los efectos debidos a la variación de las fuerzas de inercia, diferencias en la compresión y la posición de los pistones en el caso de motores de más de un cilindro [5]. En el motor de combustión interna, las vibraciones son causadas debido a las masas de movimiento alternativo y giratorio del motor. Las variaciones de las fuerzas de inercia se deben a la combustión y a las diferencias de compresión de la disposición del cilindro de pistón durante sus operaciones. Las fuerzas de inercia del motor conducen a las fuerzas desequilibradas del motor y tienden a variar con respecto a la velocidad, el suministro de combustible y las características de su combustión [2]. Las vibraciones además de ser consecuencia de movimientos mecánicos rotativos, lineales y alternantes, también tienen su origen en el proceso de combustión; por esta razón el nivel de vibración del motor depende de las propiedades del combustible como el octanaje y de la calidad de la combustión. Las vibraciones del motor dependen de su estado y de su fuente de energía, en este caso del tipo de combustible y de sus propiedades, teniendo vibraciones u oscilaciones características en sus ejes ortogonales X, Y, y Z; las mismas que pueden ser registradas por medio de los sensores de aceleración de un dispositivo de comunicación móvil [3]. Inestabilidades y defectos encontrados en los motores de combustión interna; así como también los efectos y la calidad del proceso de combustión dan lugar a un patrón de vibración distinto; por lo tanto, pueden identificarse fallos mediante técnicas de análisis del espectro de vibración [2].En la presente investigación se investiga el efecto que tiene cada uno de los combustibles utilizados en la estabilidad del motor, por medio del análisis del espectro de aceleraciones. El principio subyacente es que cada componente del motor genera vibraciones identificables. Así, el cambio en el nivel de vibración en cualquier frecuencia dada puede relacionarse directamente con las partes del motor afectadas, o con el efecto que tienen las propiedades de cada tipo de combustible [3].

\section{Materiales Y Equipo}

Los combustibles usados para la prueba fueron gasolina tipo extra y ecopaís, cada una con propiedades propias, a pesar de ser combustibles sustitutos uno del otro.

Tabla 1. Caracterización del combustible

\begin{tabular}{|c|c|c|}
\hline & Gasolina extra & Gasolina ecopaís \\
\hline Número de octano RON [-] & 88.4 & 89.3 \\
\hline Viscosidad cinemática a $20^{\circ} \mathrm{C}$ [cSt] & 0.524 & 0.532 \\
\hline Densidad API a $60^{\circ} \mathrm{F}\left[{ }^{\circ} \mathrm{API}\right]$ & 58.8 & 56.7 \\
\hline
\end{tabular}


El motor de prueba es equipado con la tecnología FLEXFUEL, la misma que permite el uso de combustibles que contengan etanol.

\begin{tabular}{ll}
\multicolumn{2}{c}{ Tabla 2. Especificación del motor } \\
\hline Motor & Otto \\
\hline Tipo & FLEXFUEL \\
Cilindrada & $3500 \mathrm{~cm}^{3}$ \\
Potencia & $302 \mathrm{hp} \mathrm{@} \mathrm{6500rpm}$ \\
Torque & 2781b·ft @ 4000rpm \\
\hline
\end{tabular}

Las aceleraciones fueron registradas con los acelerómetros de un dispositivo móvil.

Tabla 3. Especificación de los acelerómetros

\begin{tabular}{|c|c|}
\hline Resolución & $0.156 \mathrm{~m} / \mathrm{s}^{2}$ \\
\hline Rango de medición máximo & $19.6133 \mathrm{~m} / \mathrm{s}^{2}$ \\
\hline Potencia & $0.68 \mathrm{~mW}$ \\
\hline
\end{tabular}

\section{Método De La Medición}

Como primer paso, con ayuda de los acelerómetros del dispositivo de comunicación móvil, tomamos datos de las aceleraciones resultantes de las vibraciones del motor a distintas velocidades de giro con el auto en estado estacionario, para luego repetir la misma prueba con el auto en movimiento y con los dos tipos de gasolinas, para establecer el efecto que tiene cada uno de estos combustibles.

La vibración resultante del espectro de aceleración se calcula con la siguiente fórmula de la aceleración efectiva:

$$
a R M S=\sqrt{\frac{R_{1}^{2}+R_{2}^{2}+R_{3}^{2}+\cdots R_{n}^{2}}{n}}
$$

Donde:

aRMS: aceleración efectiva

$\mathrm{R}_{1}, \mathrm{R}_{2}, \mathrm{R}_{3}, \mathrm{R}_{\mathrm{n}}$ : aceleraciones resultantes

n: número de aceleraciones resultantes

Las aceleraciones resultantes fueron calculadas con cada una de las aceleraciones medidas en los ejes ortogonales $\mathrm{X}, \mathrm{Y}$ y Z .

\section{Resultados}

En condiciones estáticas, se obtuvieron los siguientes resultados de vibraciones del motor, a diferentes velocidades de giro con gasolina tipo extra. 


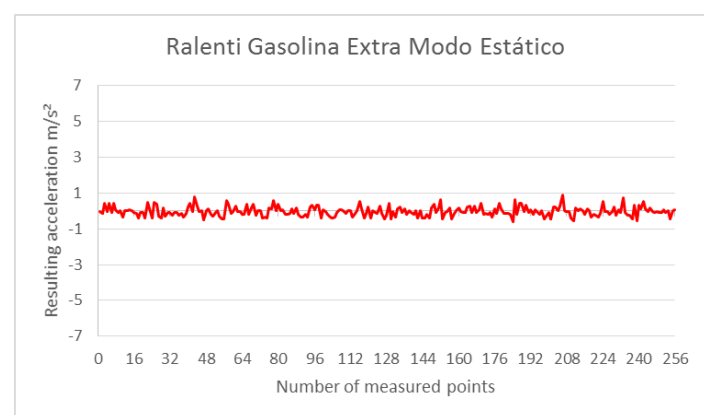

Fig. 1. Vibraciones con gasolina extra con el motor a ralentí.

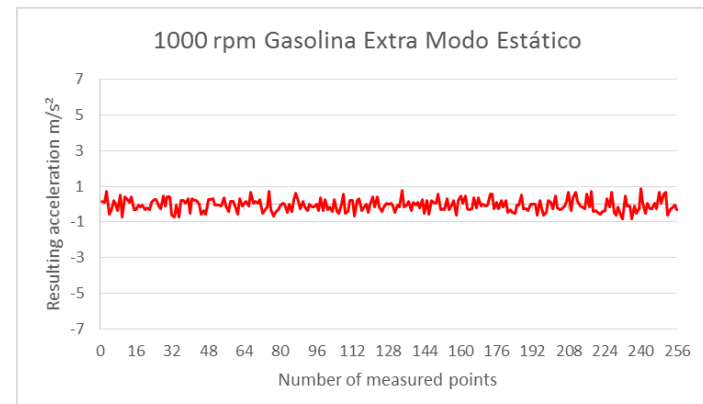

Fig. 2. Vibraciones con gasolina extra con el motor a 1000 rpm.

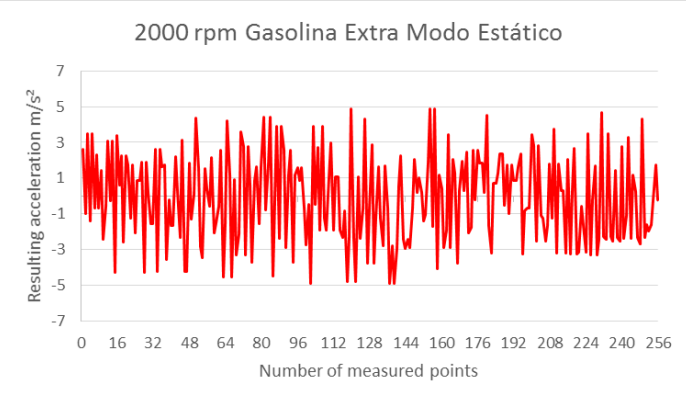

Fig. 3. Vibraciones con gasolina extra con el motor a 2000 rpm.

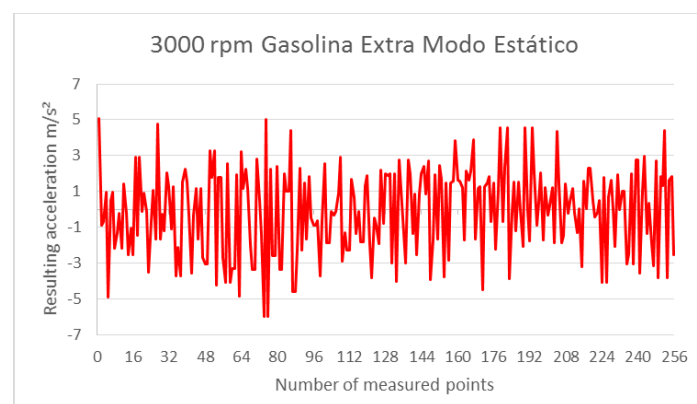

Fig. 4. Vibraciones con gasolina extra con el motor a 3000 rpm. 


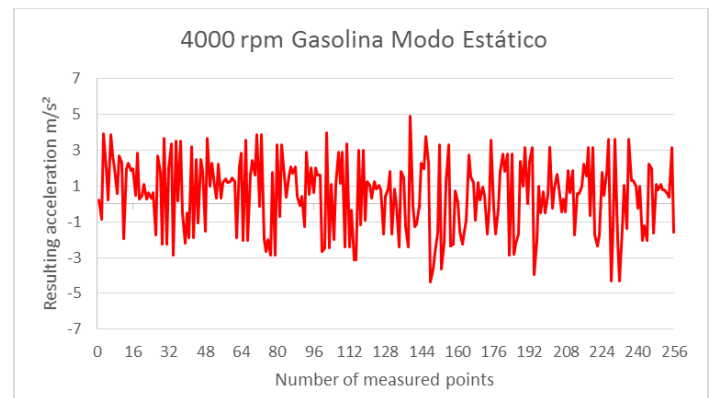

Fig. 5. Vibraciones con gasolina extra con el motor a 4000 rpm.

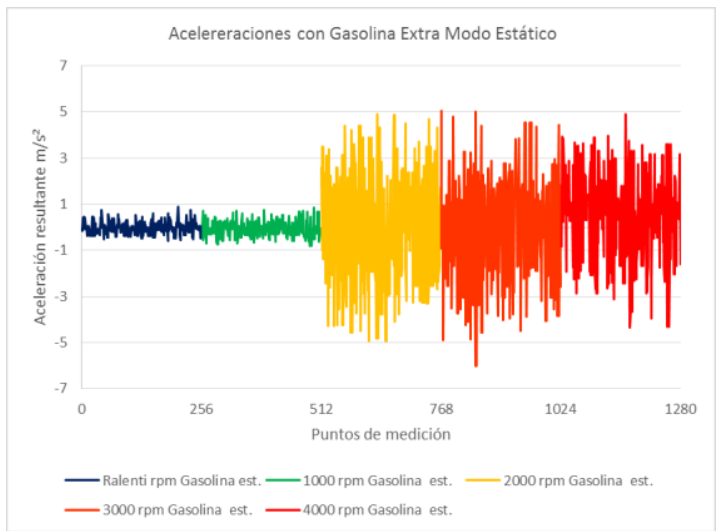

Fig. 6. Vibraciones del motor a diferentes velocidades de giro, durante la prueba estática, con gasolina extra.

Con la gasolina tipo extra, se obtuvieron los siguientes resultados de la prueba dinámica, a diferentes velocidades de giro del motor como se muestra a continuación:

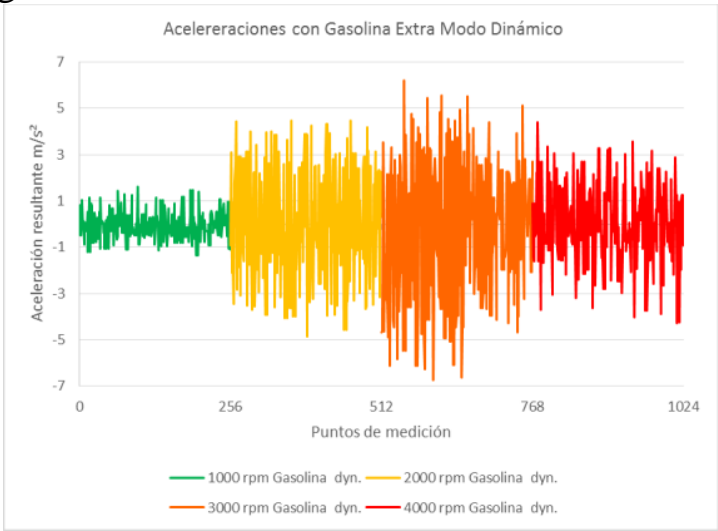

Fig. 7. Vibraciones del motor a diferentes velocidades de giro, durante la prueba dinámica, con gasolina extra.

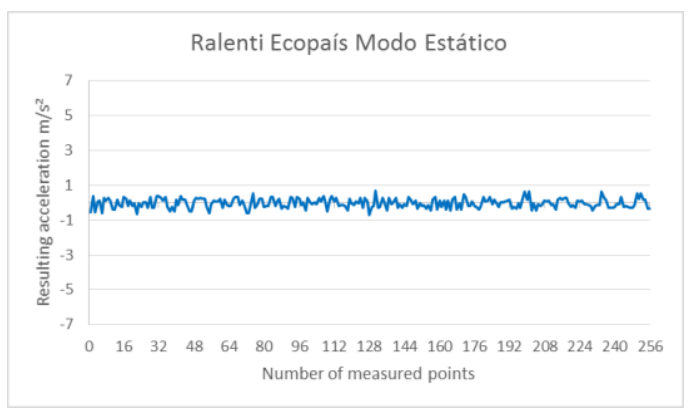


Fig. 8. Vibraciones con gasolina ecopaís con el motor a ralentí.

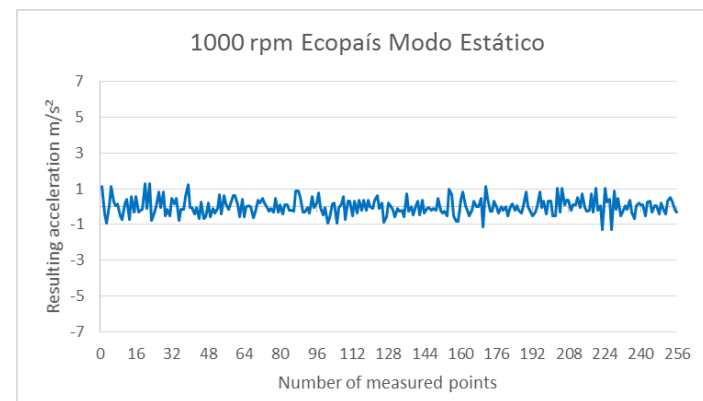

Fig. 9. Vibraciones con gasolina ecopaís con el motor a 1000 rpm.

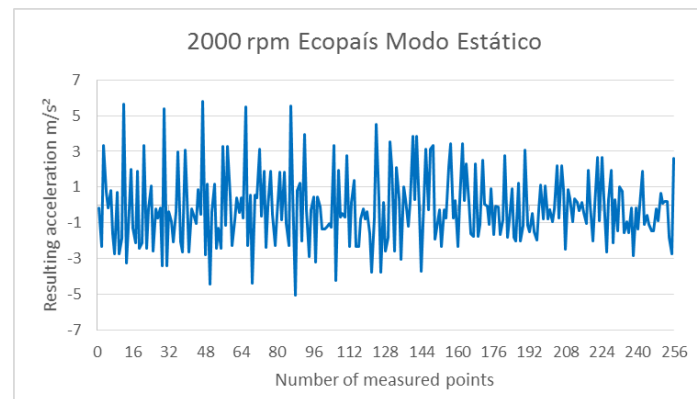

Fig. 10. Vibraciones con gasolina ecopaís con el motor a 2000 rpm.

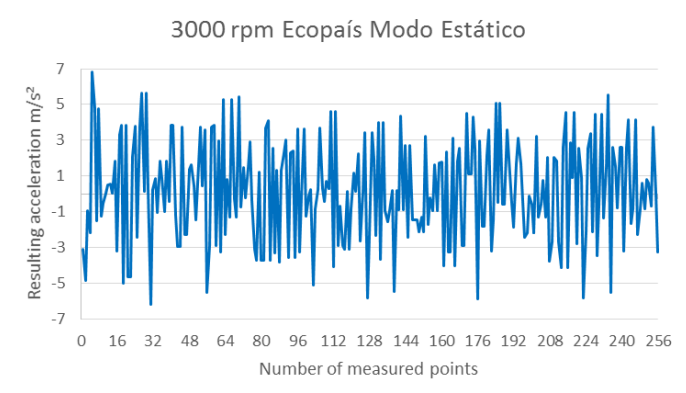

Fig. 11. Vibraciones con gasolina ecopaís con el motor a 3000 rpm.

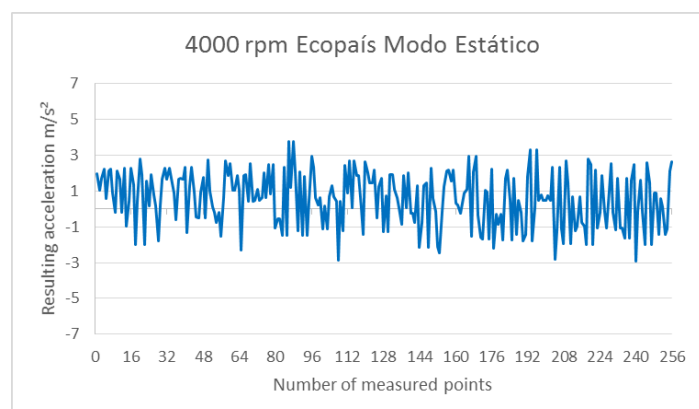

Fig. 12. Vibraciones con gasolina ecopaís con el motor a 4000 rpm. 


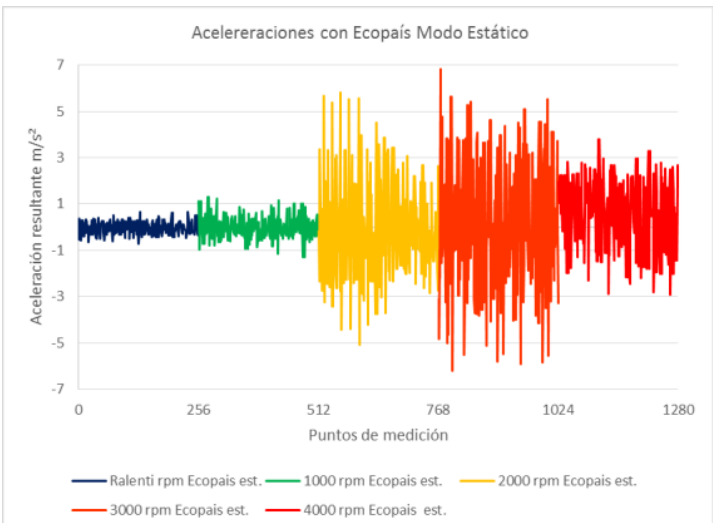

Fig. 13. Vibraciones del motor a diferentes velocidades de giro, durante la prueba estática, con gasolina ecopaís.

Los resultados de la prueba dinámica realizada con gasolina tipo ecopaís con el vehículo a diferentes velocidades de giro se muestran a continuación:

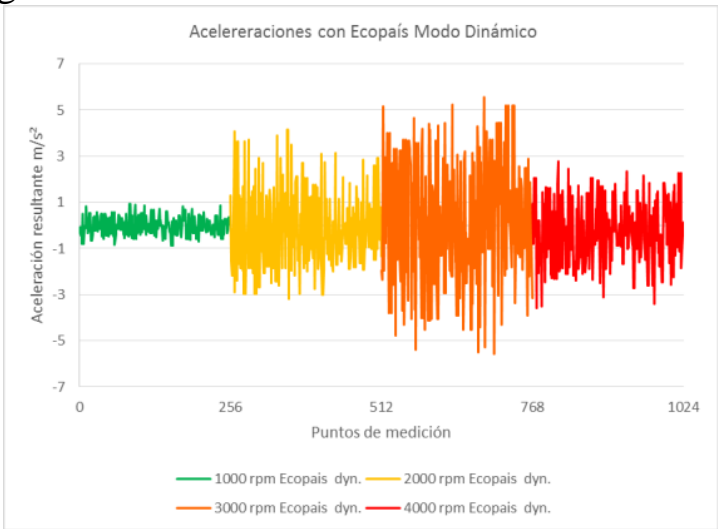

Fig. 14. Vibraciones del motor a diferentes velocidades de giro, durante la prueba dinámica, con gasolina extra.

Tabla 4. Aceleraciones RMS en $\mathrm{m} / \mathrm{s}^{2}$ calculadas.

\begin{tabular}{|c|c|c|c|}
\hline & & $\begin{array}{l}\text { Aceleraciones RMS con gasolina extra } \\
\mathrm{m} / \mathrm{s}^{2}\end{array}$ & $\begin{array}{l}\text { Aceleraciones RMS con gasolina ecopaís } \\
\mathrm{m} / \mathbf{s}^{2}\end{array}$ \\
\hline Ralentí & & 0,16 & 0,22 \\
\hline $\begin{array}{c}1000 \\
\text { estático }\end{array}$ & rpm & 0,26 & 0,31 \\
\hline $\begin{array}{l}1000 \\
\text { dinámico }\end{array}$ & rpm & 0,38 & 0,30 \\
\hline $\begin{array}{c}2000 \\
\text { estático }\end{array}$ & rpm & 2,03 & 1,19 \\
\hline $\begin{array}{l}2000 \\
\text { dinámico }\end{array}$ & rpm & 1,95 & 1,24 \\
\hline $\begin{array}{c}3000 \\
\text { estático }\end{array}$ & rpm & 1,81 & 2,30 \\
\hline $\begin{array}{l}3000 \\
\text { dinámico }\end{array}$ & rpm & 2,41 & 2,14 \\
\hline $\begin{array}{c}4000 \\
\text { estático }\end{array}$ & rpm & 1,70 & 1,41 \\
\hline $\begin{array}{l}4000 \\
\text { dinámico }\end{array}$ & rpm & 1,15 & 1,01 \\
\hline
\end{tabular}

Tabla 5. Potencia del motor con cada uno de los combustibles. 


\begin{tabular}{lll}
\hline & Gasolina Extra & Gasolina Ecopaís \\
\hline Potencia promedio [CV] & 280.2 & 297.2 \\
\hline
\end{tabular}

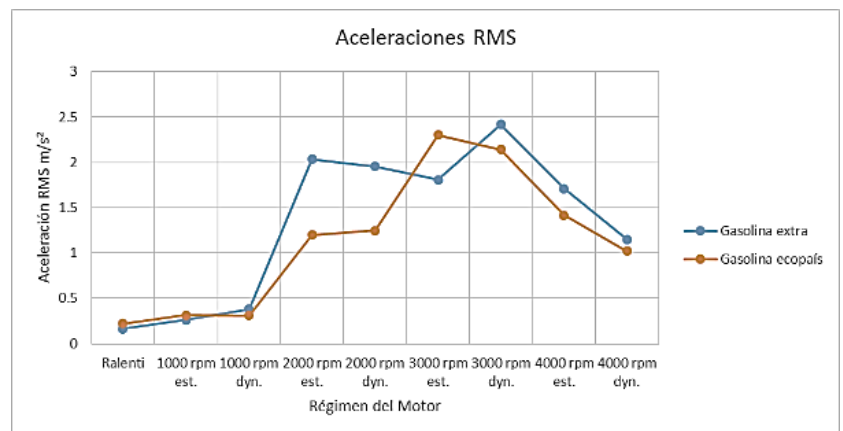

Fig. 15. Cuadro comparativo de aceleraciones RMS de gasolina Extra y Eco país.

\section{Análisis De Resultados}

Como se puede observar en las pruebas de vibraciones con gasolina tipo extra (Fig. 6 y 7), existe un mayor número de vibraciones tanto en las pruebas dinámicas como estáticas en comparación con las pruebas de vibraciones dinámicas y estática de la gasolina ecopaís (Fig. 13 y 14).

Existe una reducción de vibraciones considerable en el motor cuando se utiliza la gasolina ecopaís, en la Fig. 15 se pudo observar que la gasolina extra genera aceleraciones efectivas RMS mayores que la gasolina ecopaís.

De la tabla de caracterización se puede observar que la gasolina tipo ecopaís tiene un grado mayor de octanaje, lo que evita la detonación y mejora la calidad de la combustión. Este efecto se refleja en la reducción de las vibraciones y en el aumento de potencia.

\section{Conclusiones}

La gasolina ecopaís genera menos vibraciones en el motor de combustión interna que la gasolina extra, debido a que genera una combustión más controlada por un mejor índice de octano.

El combustible tipo ecopaís genera un incremento de potencia en el motor con respecto a la gasolina extra; ya que, al reducir la vibración del motor, la trasformación de la energía del

combustible en energía mecánica es más efectiva en su transmisión al tren de rodaje, en lugar de perderse en forma de vibraciones.

Las vibraciones del motor no solo dependen de su estado y funcionamiento mecánico sino también de la calidad del combustible y del proceso de combustión.

Se aplicó una técnica que permite evaluar la estabilidad del motor en función de las propiedades del combustible. 


\section{Bibliografía}

Asiah Ab. Rahim, Nik Rosli Abdullah. "Effect Of Alcohol-Gasoline Blends (Ethanol) On Performance And Emission Of Si Engine “. ARPN Journal of Engineering and Applied Sciences. Vol. 11, NO. 20, October, 2016.

Somashekar V., Dr. K. Satish, Jamuna AB., Ranjitha P. "Vibration Signature Analysis of IC Engine". International Journal of Innovative Research \& Development. Vol 2 Issue 13. December, 2013.

Marcos Gutiérrez, Andrés Castillo, Juan Iñiguez, Gorky Reyes. "Engine Vibrations Measurements with a Fuel Blend from Recycled Lubricating Oil and Diesel". International Powertrains, Fuels \& Lubricants Meeting. SAE Technical Paper 2017-01-2333. October, 2017.

S. Babazadeh Shayan, S. M. Seyedpour, F. Ommi, S. H. Moosavy, et al. "Impact of MethanolGasoline Fuel Blends on the Performance and Exhaust Emissions of a SI Engine". International Journal of Automotive Engineering. Vol. 1, Number 3, July 2011.

T. Ramachandran, K. P. Padmanaban. "Review On Internal Combustion Engine Vibrations And Mountings". International Journal of Engineering Sciences \& Emerging Technologies, August 2012. 